\title{
Dry Climate as a Predictor of Chagas' Disease Irregular Clusters: A Covariate Study
}

\author{
Luiz H. Duczmal*1, Gladston J. Moreira², Luís Paquete ${ }^{3}$, David Menotti², Ricardo \\ Takahashi $^{1}$ and Denise Burgarelli ${ }^{1}$
}

${ }^{1}$ Statistics, Universidade Federal de Minas Gerais, Belo Horizonte, Brazil; ²Universidade Federal de Ouro Preto, Ouro Preto, Brazil; 3Universidade de Coimbra, Coimbra, Portugal

\section{Objective}

We employ climate information to assess the possible spatial dependence on the occurrence of Chagas' disease irregular clusters in Central Brazil, using a variant of the Spatial Scan Statistic [1], the Geo-Dynamic Scan (GDScan) [4].

\section{Introduction}

Chagas' disease, caused by the protozoan Trypanosoma cruzi, is spread mostly by Triatominae bugs. High carbon dioxide emission and strong infra-red (IR) radiation are indicative of their presence. Periods of low atmospheric water saturation favor their dispersal, when the bugs' IR perception is high [2].

The Fast Subset Scan (FSScan) [3] is very efficient for the detection of the most likely geographic cluster. Covariate studies associating the presence of regular clusters with environmental factors are routinely done using the Circular Scan, the simplest version of the Spatial Scan statistic. However, if the study employs irregular clusters instead, accurate results depend on the generation of a rich family of variants of the primary cluster.

\section{Methods}

GDScan's bi-objective optimization approach simultaneously minimizes the population and maximizes the number of cases of each candidate solution. The efficient solution set of the bi-objective problem is formed by several candidate solutions, as opposed to the (generally) unique solution provided by other methods, such as the Circular Scan or the FSScan. Each candidate solution $\mathrm{z}$ is a nondominated solution, in the sense that any other solution w with more cases than $\mathrm{z}$ and smaller population than $\mathrm{z}$ cannot exist.

The idea is to penalize regions which have humid climates, since the bugs prefer dry places. Figure 1 shows Minas Gerais state in central Brazil, indicating Chagas' disease rates (Figure 1a) and dryness levels during the driest season, from June to August (Figure 1b). The efficient solution set returned by GDScan is then evaluated according to dryness value. The cluster solutions located in drier regions, considered as more plausible, should receive the higher values. For clusters with very similar likelihood ratio scores, the most plausible solution is the one with maximum dryness.

\section{Results}

Figure 1c shows GDScan's primary cluster variant with the lowest precipitation level, corresponding to the best solution found which incorporate the driest regions.

Figure $1 \mathrm{~d}$ presents variants of the primary cluster provided by GDScan (open circles) and FSScan (crosses); the "better" solutions (more cases within less populated clusters) are located in the lower-left part of the graph. As we can see, primary cluster variants generated by GDScan are better situated in the graph than those generated by FSScan.

\section{Conclusions}

The cluster of Figure 1c is nearly identical with the most likely cluster found by both GDScan and FSScan (not shown). This is an indication of a strong correlation between dryness and Chagas' disease incidence, supporting previous findings [2]. This information could be useful in Chagas' disease surveillance and prevention.

GDScan is computationally more expensive; however, it finds more potentially useful variants of the primary cluster as compared to FSScan (e.g., those variants with more desirable covariate values).
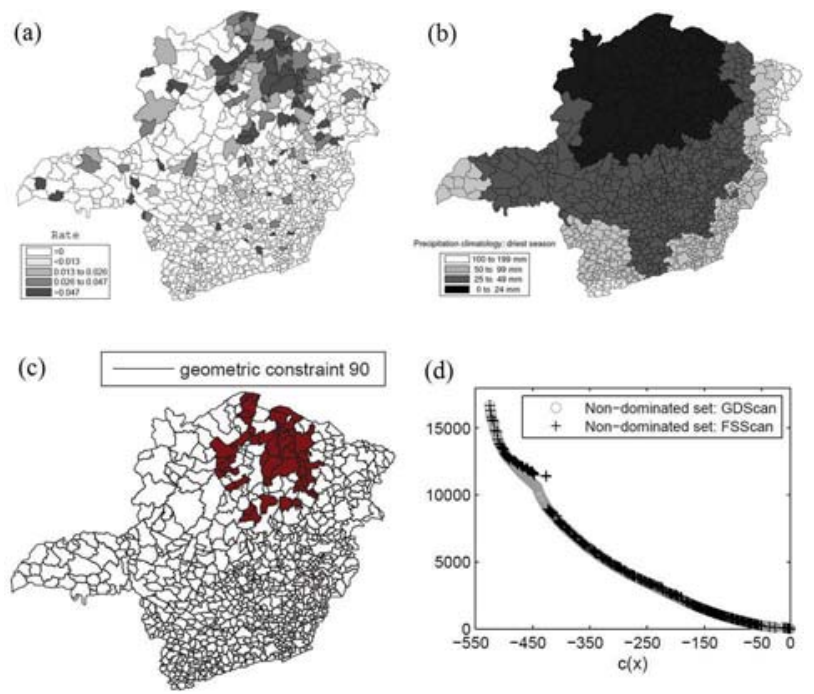

Chagas' disease rates (a), precipitation levels with darker shades indicating dryer areas (b), GDScan's primary cluster variant with lowest precipitation level (c), and variants of the primary cluster, provided by GDScan (open circles) and FSScan (crosses) (d).

\section{Keywords}

irregular cluster; spatial scan statistic; Chagas' disease; Geo Dynamic Scan; Fast Subset Scan

\section{Acknowledgments}

The authors were supported by CNPq, Fapemig and CAPES.

\section{References}

1. Kulldorff M (1997) A spatial scan statistic. Comm. Stat: Th. Meth. 26:1481-1496.

2. Catalá SS (2011) The infra-red (IR) landscape of triatoma infestans. an hypothesis about the role of IR radiation as a cue for triatominae dispersal. Infection, Genetics and Evolution 11(8):1891-1898.

3. Neill DB (2012) Fast subset scan for spatial pattern detection. JRSSB. 74:337-360.

4. Moreira GJP, Paquete L, Duczmal LH, Menotti D, Takahashi RHC (2014). Multi-objective dynamic programming for spatial cluster detection. Env. Ecol. Stat. (to appear)

\footnotetext{
*Luiz H. Duczmal

E-mail: duczmal@ufmg.br
} 